\title{
Editorial
}

Digestion

\section{The Right Colon - A Real Challenge for the Endoscopist}

\author{
J.F. Riemann \\ Klinikum Ludwigshafen, Medizinische Klinik C, Ludwigshafen am Rhein, Germany
}

There is increasing evidence (despite prospective randomized controlled trials which are still missing) that total colonoscopy may reduce the incidence of and mortality from colon cancer; however, there is a controversial debate concerning how effective colonoscopy is in relation to cancer of the proximal and the distal colon, respectively. Less cancer reduction in the proximal part compared to the distal one has been reported, but not generally confirmed. Important reasons relate to the endoscopists' skills, size and surface of lesions, the state of bowel cleaning, and the time to withdraw the endoscope. There is also an increasing body of knowledge that genetic and molecular differences may play an even more decisive role.

Obesity in the meantime has evolved as one important lifestyle risk factor for a variety of cancers including cancer of the colon. Therefore, it should be realistic to reduce adenoma recurrence after polypectomy by weight reduction. It is still an open question as to whether there are differences of adenoma recurrence in terms of location (proximal, distal or similar in both parts of the colon) in regard to demographic (age, sex) and lifestyle factors.

While the same is true for NSAIDs, which are widely used and which are generally known to reduce colon cancer, it is not known in what part of the colon and with what side effects. Information on all these data might have some influence in advising patients in terms of changing their lifestyle or in taking such drugs.
The data of the author's large prospective trial over a period of at least 4 years do not offer a real surprise. The widely observed risk factor of overweight, which ideally can be modified by a reduction of weight, did not lead to a reduction of adenoma recurrence within this time frame. As expected, NSAIDs were associated with a nearly $50 \%$ reduction on advanced adenomas in both parts of the colon, but a general recommendation at this time, especially for nonselective ones, cannot be given due to their side effects. What can we learn from this elegant study? First, that demographic factors like male sex and advanced and old age do have an influence on proximal colon adenomas, which amongst other findings is in good accordance with reports that men are at a higher risk for colon cancer compared to woman. This is important information for the general population. The endoscopist has to look extra carefully at this part of the colon, which will therefore be a major challenge for him. The findings may be in line with recent molecular findings along colon subsites, demonstrating a strong dichotomy of proximal versus distal colon [1].

Second, the assumption that modifiable lifestyle changes like reducing overweight may lead to a reduced adenoma recurrence and would therefore further contribute to colon cancer reduction is unfortunately not valid, at least not within the trial's time frame in contrast to what one might have expected. That should by no means lead to the fatal conclusion that weight reduction is worthless. In contrast, reduced body weight has other advan-

\section{KARGER}

E-Mail karger@karger.com

www.karger.com/dig (c) 2013 S. Karger AG, Basel

$0012-2823 / 13 / 0873-0139 \$ 38.00 / 0$
Prof. Dr. J.F. Riemann

Stiftung Lebensblicke

Schuckertstrasse 37

DE-67061 Ludwigshafen am Rhein (Germany)

E-Mail riemannj@garps.de 
tages in regard to cardiovascular diseases, diabetes, liver diseases, etc. As long as we do not really know whether and what kind of molecular phenomena are of any major importance and how they may be influenced, the only real and proven consequence has to be that careful colonoscopy with complete polypectomy and a clear follow-up according to the national guide lines is essential.

Once again, the key message is that thorough bowel preparation (clean colon), an exact examination by an experienced, and skilled endoscopist using high-definition endoscopes and trained in detecting and removing all the different appearances of precursor lesions (flat and/or serrated lesions) predominantly located in the proximal colon, and a standard follow-up is a condition sine qua non in colon cancer prevention. The right colon is always a real challenge for the endoscopist!

Prof. Dr. J.F. Riemann, Ludwigshafen am Rhein

\section{References}

1 Yamauchi M, Morikawa T, Kuchiba A, et al: Assessment of colorectal cancer molecular features along bowel subsites challenges the concept of distinct dichotomy of proximal versus distal colorectum. Gut $2012 ; 61 ; 847-854$ 